\title{
El inconsciente colectivo en la imagen de marca
}

\author{
Sabrine Dornelles \\ Daniel Tena" \\ Recibido: 15 de agosto \\ Aprobado: 17 de noviembre
}

\begin{abstract}
Resumen
Este artículo presenta una manera fácil de establecer qué significa una marca para los públicos. Para ello, se basa en el inconsciente colectivo fundamentado en las creencias que tenemos todos los seres humanos sobre los dioses de la mitología griega. El estudio muestra los detalles de la significación y la riqueza de las marcas en este sentido.
\end{abstract}

Palabras clave: Marca / significación / inconsciente colectivo / medición

* Este artículo es el resultado de la tesis doctoral de la investigadora Sabrine Dornelles "el perfil arquetípico de la marca", la cual obtuvo calificación cum laude y fue dirigida por Daniel Tena al interior del Grupo de investigación en Publicidad y Relaciones Públicas en la Universitat Autònoma de Barcelona.

* Forma parte del grupo de investigación GRP (Grupo de investigación en Publicidad y Relaciones Públicas) de la Universitat Autónoma de Barcelona. Participa en el Master en Comunicación Gráfica de la Universidad Autónoma de Barcelona siendo especialista de marquetinh. Correo electrónico: sdornelles@hotmail.com

*** Forma parte de los grupos de investigación GRP (Grupo de investigación en Publicidad y Relaciones Públicas), y del GRPCP (Grupo de Investigación en Psicología i Comunicación Publicitaria). Es codirector del Máster Periodismo y Comunicación Digital, programa propio organizado por la Universidad Autónoma de Barcelona y la Universidad Carlos III. También es el director del Máster en Comunicación Gráfica, programa propio de la UAB. Correo electrónico: daniel.Tena@uab.cat 


\section{The collective unconscious of the brand image}

\section{Abstract}

This article shows an easy way to establish the meaning of a brand for several audiences. For this purpose, the article is focused on the collective unconscious based on the beliefs all human beings have about the gods of the Greek mythology. The study shows all details of meaning and richness of brands in this sense.

Key words: Brand; meaning; collective unconscious; measurement. 


\section{Introducción}

Dada la importancia de las marcas comerciales en el mundo actual el presente trabajo indaga sobre la influencia que tienen los arquetipos de los dioses griegos, en tanto que referentes culturales, para establecer el significado de las marcas. Así, los arquetipos son referentes simbólicos que todas las personas tenemos y están asentados en el inconsciente colectivo de las poblaciones (Martins, 1999: 37). La presencia de patrones arquetípicos comunes en todos los pueblos se constata con las similitudes en las mitologías de distintas culturas. Como patrones preexistentes los arquetipos influencian la forma de comportamiento de cada persona (Bolen, 2006: 37-38).

Las empresas dedican grandes sumas de dinero para que su marca y su imagen de marca sean poderosas en el mercado. La construcción y la gestión de la imagen de marca deben aumentar la percepción de valor que el consumidor tiene de la marca. En este sentido la percepción es la imagen mental que el consumidor tiene de la marca. Es la personalidad de la marca que está en la mente del consumidor. De la misma manera que todas las personas tenemos una específica personalidad, las marcas también poseen su propia personalidad. Esta personalidad puede detectarse al identificar los diferentes arquetipos atribuidos a partir del inconsciente colectivo que todas las personas tenemos. En este sentido, los arquetipos son patrones comunes a todas las culturas humanas (Martins, 1999: 37).

Descubrir los arquetipos que los consumidores son capaces de identificar en las imágenes de marca responde a la relación entre la marca y el consumidor. La descripción del Perfil Arquetípico de la Marca nos permitirá estar en condiciones de construir una marca fuerte y gestionar adecuadamente su imagen de marca. Y, finalmente conectar con los consumidores de una forma más profunda y emocional.
Si hablamos de marcas, hoy en día, el contexto en el que vivimos es como, Lee Clow, presidente de TBWA Worldwide y desarrollador creativo de la campaña de Apple "think Differente" ("Piensa Diferente") explica: Las marcas ya no son sólo la manera de recordarte lo que tú quieres comprar. Se han convertido en parte del tejido social de nuestra sociedad. Las marcas forman parte de nuestro sistema de ordenación de las cosas; incluso crean el contexto en el que podemos ser y vivir como personas... las marcas, hoy en día, articulan quién eres y te caracterizan (Clifton y Maughan, 2000: 71). Una marca ya no es un signo monótono utilizado para identificar a una organización, un logotipo bidimensional que pueda pegarse en el exterior de una botella. En la actualidad, las marcas funcionan como señales que denotan y connotan identidad humana (Atkin, 2005:139). Por tanto, una marca está destinada a desarrollar un perfil de personalidad de cara al consumidor.

Para abordar el inconsciente colectivo debemos hacer mención de Carl Gustav Jung (18751961), fundador de la psicología analítica. Él es el creador de los conceptos de inconsciente colectivo y de arquetipos. Según Jung el inconsciente representa una condición que está antes de la conciencia y de sus contenidos (Jung, 1991: 7). Advierte que el inconsciente no es lo simplemente desconocido. Puede ser definido como una realidad extremadamente fluctuante: "todo lo que uno sabe, pero en lo cual momentáneamente no piensa; todo lo que alguna vez fue consciente, pero que ahora uno ha olvidado; todo lo percibido por los sentidos, pero que la consciencia no advierte; todo lo que, sin intención ni atención, es decir, inconscientemente, uno siente, piensa, recuerda..." (Jung, 1991:7- 9). Existe una diferencia básica entre el inconsciente colectivo y el inconsciente personal. El inconsciente personal es más superficial y se origina en la experiencia y la adquisición personal, explica Jung (1991: 9-10). El inconsciente colectivo es innato, no es de naturaleza individual sino universal. Es decir, es lo mismo en todos los hombres y mujeres. 
Sólo podemos hablar de un inconsciente cuando es posible verificar la existencia de contenidos del mismo. Los contenidos del inconsciente personal son en lo fundamental los llamados complejos de carga afectiva, que forman parte de la intimidad de la vida anímica. En cambio, los contenidos del inconsciente colectivo son lo que entendemos por arquetipos (Jung, 1991:10) '.

Los arquetipos señalan vías determinadas a todas las actividades de la fantasía y los mitos. Así como los arquetipos aparecen como mitos en las historias de los pueblos, también se encuentran en cada individuo y ejercen su influencia más intensa. En este sentido, Jung resalta (1991: 77):

"El arquetipo en sí forma parte de los más elevados valores del alma humana y ha poblado por ello todos los Olimpos de todas las religiones. Apartarlo a un lado como algo sin valor, constituye una positiva pérdida. La tarea a realizar consiste en lo contrario, en disolver esas proyecciones para hacer que sus contenidos vuelvan a quien, por una exteriorización espontánea, los ha perdido."

Los mitos y los cuentos de hadas son expresiones de los arquetipos, como también lo son muchas imágenes y temas de los sueños. La presencia de patrones arquetípicos comunes en todos los pueblos constata las similitudes en las mitologías de distintas culturas. Como patrones preexistentes, los arquetipos influencian la forma de comportamiento de las personas (Bolen 2006: 37-38).

De acuerdo con la teoría junguiana, las diosas y los dioses son arquetipos, fuentes de patrones emocionales de los pensamientos, sentimientos, instintos y comportamiento.

1 Es lo mismo que sugiere Rapaille (2006: 27): "The principles cannot be ascribed to either the Freudian individual unconscious that guides each of us as members of the human race. The principles illuminate an unconscious that uniquely guides each of us depending on the cultures that produced us. The third unconscious is the cultural unconscious".

\section{Metodología}

Nuestro trabajo sobre la existencia e influencia de los referentes culturales en las marcas se inició con la declaración de las 4 hipótesis siguientes:

$\mathrm{H}_{1}$ : existe relación entre arquetipos y marcas.

$\mathrm{H}$ : podemos identificar al menos un arquetipo en las marcas convencionales.

$\mathrm{H}$ : podemos identificar más de un arquetipo en las ${ }^{3}$ marcas convencionales.

$\mathrm{H}$ : podemos construir el perfil arquetípico de las ${ }^{4}$ marcas.

El siguiente paso fue la fundamentación teórica basada en la metodología cualitativa para acabar, finalmente, en la metodología cuantitativa de orientación experimental.

Los mitos de los dioses del Olimpo tenían atributos muy humanos: su conducta, reacciones emocionales, la apariencia y la mitología proporcionan patrones que se asemejan a la conducta y actitudes humanas. También son familiares porque son arquetipos; es decir, representan modelos colectivos que la sociedad comparte. Los más famosos de ellos eran los quince dioses del Olimpo: ocho dioses -Zeus, Poseidón, Hades, Apolo, Ares, Hefestos, Hermes, Dionisio- y siete diosas -Hestia, Deméter, Perséfone, Hera, Artemisa, Atenea, Afrodita-. Los dioses griegos son imágenes de hombres y mujeres que viven en la imaginación humana desde hace más de tres mil años. Son patrones o representaciones de cómo son las personas. Ellos son caracterizados por patrones inherentes, que en definitiva, son representados por los arquetipos. Los dioses difieren unos de otros. Cada uno de ellos tiene trazos positivos y otros potencialmente negativos. Sus mitos muestran lo que es importante para ellos y se expresan por metáforas (Bolen 2006: 26-50). 
Tabla 1. Los arquetipos analizados: simbolizan las siete diosas griegas y los ocho dioses griegos.

\begin{tabular}{|l|l|}
\hline \multicolumn{1}{|c|}{ Diosas } & \multicolumn{1}{|c|}{ Dioses } \\
\hline Afrodita & Apolo \\
Artemisa & Ares \\
Atenea & Dionisio \\
Deméter & Hades \\
Hera & Hefesto \\
Hestia & Hermes \\
Perséfone & Poseidón \\
& Zeus \\
\hline
\end{tabular}

Fuente: Sabrine Dornelles, GRP 2010

Para crear el cuadro de los arquetipos nos basamos en las características que mejor describieran y discriminasen el perfil de cada arquetipo. Partiendo de las características que explican cada arquetipo fueron seleccionadas las tres características principales, que se identificaron como evidentes en la revisión bibliográfica, para cada uno de los arquetipos.

Tabla 2. Arquetipos y palabras clave obtenidas a partir de la frecuencia de citación entre los autores de referencia.

\begin{tabular}{|c|c|}
\hline Arquetipo & Palabra clave \\
\hline Adolescente & $\begin{array}{l}\text { Juventud } \\
\text { Rebeldía } \\
\text { Sociabilidad }\end{array}$ \\
\hline Artista & $\begin{array}{l}\text { Emoción } \\
\text { Pasión } \\
\text { Sensibilidad }\end{array}$ \\
\hline Celebridad & $\begin{array}{l}\text { Atractividad } \\
\text { Seducción } \\
\text { Sensualidad }\end{array}$ \\
\hline Esposa & $\begin{array}{l}\text { Conservadorismo } \\
\text { Compromiso } \\
\text { Tradición }\end{array}$ \\
\hline Explorador & $\begin{array}{l}\text { Aventura } \\
\text { Dinamismo } \\
\text { Independencia }\end{array}$ \\
\hline
\end{tabular}

\begin{tabular}{|c|c|}
\hline Arquetipo & Palabra clave \\
\hline Héroe & $\begin{array}{l}\text { Competición } \\
\text { Fuerza } \\
\text { Superación }\end{array}$ \\
\hline Intelectual & $\begin{array}{l}\text { Inteligencia } \\
\text { Objetividad } \\
\text { Racionalidad }\end{array}$ \\
\hline Inventor & $\begin{array}{l}\text { Creatividad } \\
\text { Funcionalidad } \\
\text { Innovación }\end{array}$ \\
\hline Madre & $\begin{array}{l}\text { Amabilidad } \\
\text { Maternal } \\
\text { Protección }\end{array}$ \\
\hline Niño & $\begin{array}{l}\text { Diversión } \\
\text { Lúdico } \\
\text { Travesura }\end{array}$ \\
\hline Patriarca & $\begin{array}{l}\text { Autoridad } \\
\text { Poder } \\
\text { Respeto }\end{array}$ \\
\hline Princesa & $\begin{array}{l}\text { Alegría } \\
\text { Inocencia } \\
\text { Romanticismo }\end{array}$ \\
\hline Príncipe & $\begin{array}{l}\text { Elegancia } \\
\text { Equilibrio } \\
\text { Estética }\end{array}$ \\
\hline Voluntario & $\begin{array}{l}\text { Caritativo } \\
\text { Generosidad } \\
\text { Humanitaria }\end{array}$ \\
\hline Zen & $\begin{array}{l}\text { Ecológico } \\
\text { Natural } \\
\text { Serenidad }\end{array}$ \\
\hline
\end{tabular}

Fuente: Sabrine Dornelles, GRP 2010

A partir de esta asignación, se abordó la investigación desde la perspectiva experimental. Para ello, el test de recepción se realizó con una muestra aleatoria no probabilística, que según Hernández et alt. (2003: 326) es un procedimiento de selección informal; según este tipo de muestra no se busca la correlación de los sujetos para una población sino la correlación entre las variables. Utilizaremos una muestra de conveniencia; en esta muestra se elige un grupo existente designado 
como muestra. Este es un método fácil y barato aunque el sesgo suele ser imposible de estimar. Utilizamos el modelo de ANOVA de factor con medidas repetidas que según Igártua consiste en medir más de una variable en una misma muestra de sujetos. Se trataba de identificar si podíamos identificar los valores en referencia al inconsciente colectivo. Para ello se analizaron 3 marcas comerciales (Nike, Adidas y Puma) que son consideradas las variables independientes y un grupo de sujetos experimentales que opinaron sobre las tres marcas. De esta forma, necesitamos menos sujetos y eliminamos la variación residual que hay generalmente en la diferencia de los sujetos si hubiéramos utilizado una muestra con un grupo de sujetos para cada marca en la investigación. Así pues, utilizamos el mismo grupo de sujetos para las 3 marcas. Este procedimiento es considerado un muestreo de conveniencia en el que se analizan aquellos contenidos que están disponibles por parte del investigador (Igártua, 2006: 357).

Gráfica 1. Para el desarrollo de la experimentación se utilizaron las marcas Nike, Adidas y Puma

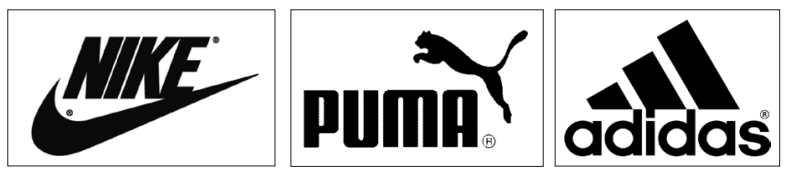

Fuente: http://www.visual.gi/logoteca/logos.html [Fecha de consulta en 22 de octubre de 2009|.

\section{Resultados}

La presente investigación tenía como finalidad conocer la relación entre los arquetipos y las marcas con la finalidad de establecer la presencia del inconsciente colectivo de las marcas. No es, por tanto, una investigación poblacional sino que tiene un componente experimental al relacionar las variables formales que participan en el proceso de comunicación, y lo es, al tomar como referente de la medición a los sujetos experimentales.

Para determinar el grado de confiabilidad ${ }^{2}$ del instrumento que hemos desarrollado para este estudio utilizaremos dos métodos de consistencia interna. Se basan en obtener coeficientes de consistencia de los datos recogidos en los diferentes tests utilizados. Así que utilizaremos el coeficiente de Kuder-Richardson (específicamente el que se conoce como fórmula 20) que se usa para tests con ítems dicotómicos (Kuder, E Richardson , 1937: 151 160) y el coeficiente alfa de Cronbach, aplicable a escalas de varios valores posibles.

2 Confiabilidad.- Bajo la denominación genérica se agrupan todo un conjunto de métodos y técnicas utilizadas por los investigadores para estimar el grado de precisión con el que está midiendo sus variables; la "confiabilidad" indica la consistencia del proceso de medición de los resultados (Bonilla, 2006: 1).

Tabla 3. Matriz experimental

\begin{tabular}{|l|l|}
\hline Variables independientes consideradas (VIC) & 3 marcas (NIKE, ADIDAS y PUMA) \\
\hline Combinaciones de las marcas & 6 \\
\hline Valores posibles en cada variable (VV) & 2: Sí, NO \\
\hline Arquetipos relacionados & 15 \\
\hline \multirow{2}{*}{ Sujetos experimentales (SE) } & 630 SE \\
& $\begin{array}{l}6 \text { combinaciones x 2 valores de la variable x 20 sujetos por } \\
\text { valor variable mínimo }=240 \text { SE necesario como mínimo) }\end{array}$ \\
\hline Fecha de aplicación & $\begin{array}{l}\text { Las entrevistas se realizaron en la Universidad Autónoma de } \\
\text { Barcelona en los días 12, 13, 16 17 e 18 de noviembre de 2009 } \\
\text { de las 9h hasta las 21h. Los cuestionarios fuero aplicados } \\
\text { por 2 investigadores. }\end{array}$ \\
\hline
\end{tabular}

Fuente: Sabrine Dornelles, GRP 2010 
Gráfica 2. Instrumento de medición.

1.1 Por favor, indica a continuación los siguientes datos.

\begin{tabular}{|l|l|l|}
\hline \multicolumn{2}{|c|}{ Sexo } & Edad \\
\hline F & $M$ & \\
\hline
\end{tabular}

\begin{tabular}{|c|c|}
\hline Carrera & \\
\hline Curso & \\
\hline
\end{tabular}

\begin{tabular}{|l|c|c|c|}
\hline \multicolumn{3}{|c|}{ Contesta a las respuestas afirmativas colocando una "X" en la casilla correspondiente } \\
\hline & Adidas & Nike & Puma \\
\hline ¿Conoces la marca? & & & \\
\hline ¿Has consumido o consumes la marca? & & & \\
\hline ¿Te gusta o gustaría consumir la marca? & & & \\
\hline
\end{tabular}

Indica con una "X" en la casilla corespondiente al SI o al NO, si consideras presentes los atributos asociados con cada marca. No hay respuestas buenas o malas, sólo deseamos saber su opinión.

\begin{tabular}{|c|c|c|c|}
\hline Marca & Atributos & Sí están presentes & NO están presentes \\
\hline & $\begin{array}{c}\text { Juventud } \\
\text { Rebeldía } \\
\text { Sociabilidad }\end{array}$ & & \\
\hline \\
\hline
\end{tabular}

La consistencia, precisión o estabilidad temporal del proceso de mediación suele expresarse mediante estos dos coeficiente de confiabilidad que varía entre 0 a 1 . Cuanto más próximo el coeficiente está de 1 más precisión y confiabilidad tiene el proceso de medición. La media utilizada por los autores es de más que 0,5, y 0,6 ya puede ser considerado aceptable. Pero cuanto más próximo de 1 más fiable es el instrumento. Según George y Mallery (1995:47), el alfa de Cronbach y el Kunder-Richardson por debajo de 0,5 muestra un nivel de fiabilidad no aceptable; si tomara un valor mayor que 0,5 y 0,6 se podría considerar como un nivel aceptable; en el intervalo 0,8-0,9 se podría calificar como de un nivel bueno, y si tomara un valor superior a 0,9 sería excelente. Kuder-Richardson.- Kuder y Richardson (1937) desarrollaron varios modelos para estimar la confiabilidad de consistencia interna de una prueba; uno de los más conocidos es la denominada fórmula 20. El modelo de Kuder-Richardson es aplicable en las pruebas de ítem es dicotómicos en los cuales existen respuestas correctas e incorrectas. Esta técnica se basa en el supuesto de que cada ítem del instrumento constituye una prueba paralela, de modo que cada ítem es tratado como paralelo de todos los demás ítems. Esta técnica solo es aplicable en aquellos casos en que las respuestas 
a cada ítem puede calificarse como 1 ó 0 cada una (correcto-incorrecto, presente- ausente, a favor-en contra, etc.). Así que el Kr20 se adapta más a nuestro caso ya que las respuestas en cada uno de las variables era SÍ o NO.

Para determinar el coeficiente Kuder-Richardson hemos calculado la varianza de cada una de las variables, es decir de cada arquetipo, luego hemos calculado la suma de las varianzas. Para calcular tanto la varianza como la suma de las varianzas hemos utilizando el programa estadístico SSPS Statistics (PAWS 18). Y finalmente se aplicó la fórmula para determinar el Kr20.

Gráfica 3. Fórmula utilizada para determinar el Kr 20. $\mathrm{K}=$ numero de Ítems; $\sigma=$ varianza.

$$
K r 20=\frac{K}{K-1}\left\{1-\left\{\frac{\sum \sigma^{2}}{\sigma^{2}}\right\}\right\}
$$

Los resultados de esta prueba han oscilado entre $\mathrm{Kr} 20=0,972$ y r20 $=0.992$. Eso nos indica que el instrumento utilizado para esta investigación tiene una alto grado de fiabilidad dado que los valores de Kuder-Richardson están muy cerca del valor 1 .

También hemos calculado el Alfa de Cronbach $^{3}$ para determinar la fiabilidad del instrumento que hemos utilizado para esta investigación. Utilizamos el coeficiente Alfa de Cronbach como un refuerzo de fiabilidad de nuestro instrumento, como un apoyo al los resultados coeficiente Kunder-Richardson aun que se considera más indicado para este estudio el Kr20 como hemos explicado anteriormente al tratarse de una investigación donde los ítems son considerados

3 El coeficiente Alfa de Cronbach es más utilizado en los casos de la medición de constructos a través de escalas, en los que no existen respuestas correctas ni incorrectas, sino que cada sujeto marca el valor de la escala que mejor representa su respuesta, Cronbach (1951) derivó, a partir del modelo de Kuder- Richardson (1937), una variante que permite estimar la confiabilidad de consistencia interna en estos casos. por respuestas dicotómicas (Sí o NO). El Alfa de Cronbach también se trata de un índice de consistencia interna que toma valores entre 0 a 1 y que sirve para comprobar si el instrumento que se está evaluando recopila información defectuosa y, por tanto, nos llevaría a conclusiones equivocadas o si se trata de un instrumento fiable que hace mediciones estables y consistentes. Cuanto más próximo a 1 más fiable es el instrumento. Para encontrar el coeficiente Alfa de Cronbach hemos utilizado directamente el programa informático SPSS Statistics (PAWS 18). En este caso el Alfa de Cronbach a oscilado entre $\alpha=0,679$ y $\alpha=0,729$. Por ello que podemos considerar el instrumento fiable.

Una vez seguros de que el instrumento utilizado es válido, pasamos al análisis de las variables estudiadas (arquetipos). Para ello recurrimos a dos importantes pruebas estadísticas que se llaman el chi-cuadrado $(\chi 2)$ y la t de Student.

La prueba del Chi-cuadrado $(\chi 2)$ es considerada como una prueba no paramétrica que mide la discrepancia entre una distribución observada y otra teórica, indicando en qué medida las diferencias existentes entre ambas, de haberlas, se deben al azar en el contraste de hipótesis. Según Igartua (2006:528):

"El contraste $\mathrm{x}^{2}$ permite decidir si dos variables son estadísticamente independientes (hipótesis nula) o si, por el contrario existe entre ellas alguna asociación (Hipótesis alternativa)".

La prueba t de Student también es una prueba no paramétrica que tiene como objetivo encontrar la diferencia entre proporciones o porcentajes. La prueba t de Student es quizá la prueba más universal para la comparación de dos tratamientos experimentales (Igartua, 2006:540).

Con estos datos podremos definir si existe relación significativa entre los arquetipos y las marcas, y cuáles son los arquetipos que los en- 
cuestados han determinado que están presentes en cada una de las marcas. Además, también utilizamos estos datos para el contraste de hipótesis. Es decir, para rechazar o no la hipótesis nula. Para definir el chi-cuadrado y la T de Student hemos utilizado el programa informático SPSS Statistics (PASW 18.0) y hemos utilizado los valores P $\leq$ 0,05 para el chi-cuadrado. Cuanto mayor sea el valor de $\chi 2$, menos verosímil es que la hipótesis sea correcta. De la misma forma, cuanto más se aproxima a cero el valor de chi-cuadrado, más ajustadas están ambas distribuciones. O sea, si este valor es menor o igual a 0,05 rechazamos la hipótesis nula y consideramos que hay significación y podremos afirmar que el arquetipo está presente en la marca. Los valores más grande que 0,05 son considerados no significativos así que no se rechaza la hipótesis nula. En este caso deberemos considerar que el arquetipo no está presente en la marca.

El nivel de significación de un test es un concepto estadístico asociado a la verificación de una hipótesis. En pocas palabras, se define como la probabilidad de tomar la decisión de rechazar la hipótesis nula cuando es verdadera (decisión conocida como error de Tipo I, o "falso positivo"). La decisión se toma a menudo utilizando el valor $\mathrm{P}$ (o p-valor): si el valor P es inferior al nivel de significación, entonces la hipótesis nula es rechazada. Cuanto menor sea el valor P, más significativo será el resultado.

$\mathrm{P} \leq 0,05$ Diferencia significativa en la variable así que se rechaza la hipótesis nula.

$\mathrm{P}>0,05$ Diferencia no significativa en la variable, así que no se rechaza la hipótesis nula.

Por otra parte, en estadística, un resultado se denomina estadísticamente significativo cuando no es probable que haya sido debido al azar. Una "diferencia estadísticamente significativa" solamente significa que hay evidencias estadísticas de que hay una diferencia; no significa que la diferencia sea grande, importante, o significativa en el sentido estricto de la palabra. Por eso después de definir los arquetipos que son significativos en la marca, analizaremos la frecuencia y el porcentaje para determinar cuáles de los arquetipos significativos son dominantes, latentes y regresivos. Es decir, para nosotros los arquetipos que son significativos serán aquellos que presenten un porcentaje y una frecuencia alta. Para ello, lo haremos en función a los valores de la tabla 4.

Tabla 4. Datos para clasificación de los arquetipos.

\begin{tabular}{|l|c|c|}
\hline \multicolumn{1}{|c|}{ Arquetipo } & Frecuencia (SI) & Porcentaje \\
\hline Dominante & $\mathrm{p} \geq 32$ & $\mathrm{p} \geq 75 \%$ \\
\hline Latente & $11>\mathrm{p}<31$ & $25 \%>\mathrm{p}<75 \%$ \\
\hline Regresivo & $\mathrm{p} \leq 11$ & $\mathrm{p} \leq 25 \%$ \\
\hline
\end{tabular}

Fuente: Sabrine Dornelles, GRP 2010

Los valores de la tabla nos permiten determinar cuándo los arquetipos serán considerados dominantes, latentes o regresivos. Los dominantes serán aquellos en los que el porcentaje de respuestas afirmativas sea igual o superior a $75 \%$ o con una frecuencia de superior o igual a 31 casos, es decir, que si 31 o más sujetos han contestado que sí el arquetipo está presente en la marca. Los arquetipos que obtengan una frecuencia de Si entre 10 y 31 , o sea de $25 \%$ a $75 \%$ serán considerados arquetipos latentes. Y por último, los arquetipos regresivos serán aquellos que su frecuencia y su porcentaje sea menor que 10 y $25 \%$, respectivamente.

Partiendo de este análisis definimos los arquetipos considerados significativos (dominantes, latentes y regresivos) de cada una de las marcas, lo que nos permite ya definir el perfil arquetípico de la Marca.

Como podemos observar los arquetipos considerados significativos en el caso ADIDAS son los que el $X^{2}(p)$ es menor o igual a 0,005. 
Según los datos de la tabla 5 podemos observar que el chi-cuadrado es menor o igual a 0,05 en los arquetipos artista, esposa, inventor, princesa, voluntario y zen. Los demás arquetipos no parecen ser significativos, ya que posee un $\mathrm{x}^{2}$ más grande que 0,05.

La t de Student da un valor significativo de 0,00 para todos los arquetipos, es decir, para todas las variables. Esto quiere decir que todos los arquetipos son significativos. Así que en este caso utilizaremos los resultados de la $\mathrm{x}^{2}$ para considerar las variables que son significativas o no. Y la t de Student solamente utilizaremos como una referencia que reafirma los valores del chi-cuadrado. Como hemos indicado en la tabla 5 los arquetipos significativos en la marca Adidas son los arquetipos artista, esposa, inventor, princesa, voluntario y zen. Partiendo de estos arquetipos significativos encontrados en la marca Adidas con los valores del chi-cuadrado, determinaremos los arquetipos dominantes y regresivos utilizando la frecuencia y el porcentaje.

Tabla 5. Chi-cuadrado y t de Student de la marca ADIDAS. Resumen de los datos de contraste de hipótesis obtenidos a partir de la información aportada por los sujetos experimentales. El nivel de significación utilizado en todos los contrastes es para el 0,5. t de Student y 0,05 para el chi-cuadrado.

\begin{tabular}{|c|c|c|}
\hline \multicolumn{3}{|c|}{ Adidas } \\
\hline Arquetipo & Chi-cuadrado & $t$ de student \\
\hline Artista & $x 2=7,714 \quad p=0,005$ & $\mathrm{t}=17,211 \quad \mathrm{p}=, 000$ \\
\hline Celebridad & $x 2=2,381 \quad p=0,123$ & $\mathrm{t}=14,755 \mathrm{p}=, 000$ \\
\hline Intelectual & $\mathrm{x} 2=3,429 \quad \mathrm{p}=0,064$ & $\mathrm{t}=15,272 \mathrm{p}=, 000$ \\
\hline Esposa & $\mathrm{x} 2=21,429 \quad \mathrm{p}=0,000$ & $t=11,763 \quad p=, 000$ \\
\hline Explorador & $x 2=3,429 \quad p=0,064$ & $\mathrm{t}=11,450 \quad \mathrm{p}=, 000$ \\
\hline Héroe & $x 2=4,667 \quad p=0,031$ & $t=16,489 \quad p=, 000$ \\
\hline Madre & $x 2=6,095 \quad p=0,014$ & $\mathrm{t}=11,319 \mathrm{p}=, 000$ \\
\hline Inventor & $x 2=7,714 \quad p=0,005$ & $\mathrm{t}=11,137 \quad \mathrm{p}=, 000$ \\
\hline Patriarca & $x 2=3,429 \quad p=0,064$ & $\mathrm{t}=11,454 \mathrm{p}=, 000$ \\
\hline Niño & $\mathrm{x} 2=0,857 \quad \mathrm{p}=0,355$ & $\mathrm{t}=12,015 \quad \mathrm{p}=, 000$ \\
\hline Princesa & $\mathrm{x} 2=21,429 \quad \mathrm{p}=0,000$ & $\mathrm{t}=24,834 \mathrm{p}=, 000$ \\
\hline Príncipe & $x 2=2,381 \quad p=0,123$ & $\mathrm{t}=14,755 \mathrm{p}=, 000$ \\
\hline Voluntario & $\mathrm{x} 2=16,095 \mathrm{p}=0,000$ & $\mathrm{t}=21,354 \mathrm{p}=, 000$ \\
\hline Zen & $\mathrm{x} 2=13,714 \quad \mathrm{p}=0,000$ & $\mathrm{t}=20,064 \quad \mathrm{p}=, 000$ \\
\hline
\end{tabular}

Fuente: Sabrine Dornelles, GRP 2010

Tabla 6. Arquetipos Dominantes y Regresivos Adidas. Resumen de los datos de "estos arquetipo está presente en la marca Adidas" obtenidos a partir de la información aportada por los sujetos experimentales.

\begin{tabular}{|l|c|c|c|l|}
\hline \multicolumn{5}{|c|}{ Adidas } \\
\hline \multicolumn{1}{|c|}{ Arquetipos } & $\chi^{2}$ & Frecuencia (SI) & $\%$ & \multicolumn{1}{|c|}{ Clasificación } \\
\hline Esposa & $\mathrm{p}=0,005$ & 36 & 85,7 & Dominante \\
Inventor & $\mathrm{p}=0,000$ & 30 & 71,4 & Latente \\
Artista & $\mathrm{p}=0,005$ & 12 & 28,6 & Latente \\
Zen & $\mathrm{p}=0,000$ & 9 & 21,4 & Regresivo \\
Voluntario & $\mathrm{p}=0,000$ & 8 & 19 & Regresivo \\
Princesa & $\mathrm{p}=0,000$ & 6 & 14,3 & Regresivo \\
\hline
\end{tabular}

Fuente: Sabrine Dornelles, GRP 2010 
Gráfica 4. Arquetipos Dominantes, Latentes y Regresivos de Adidas.

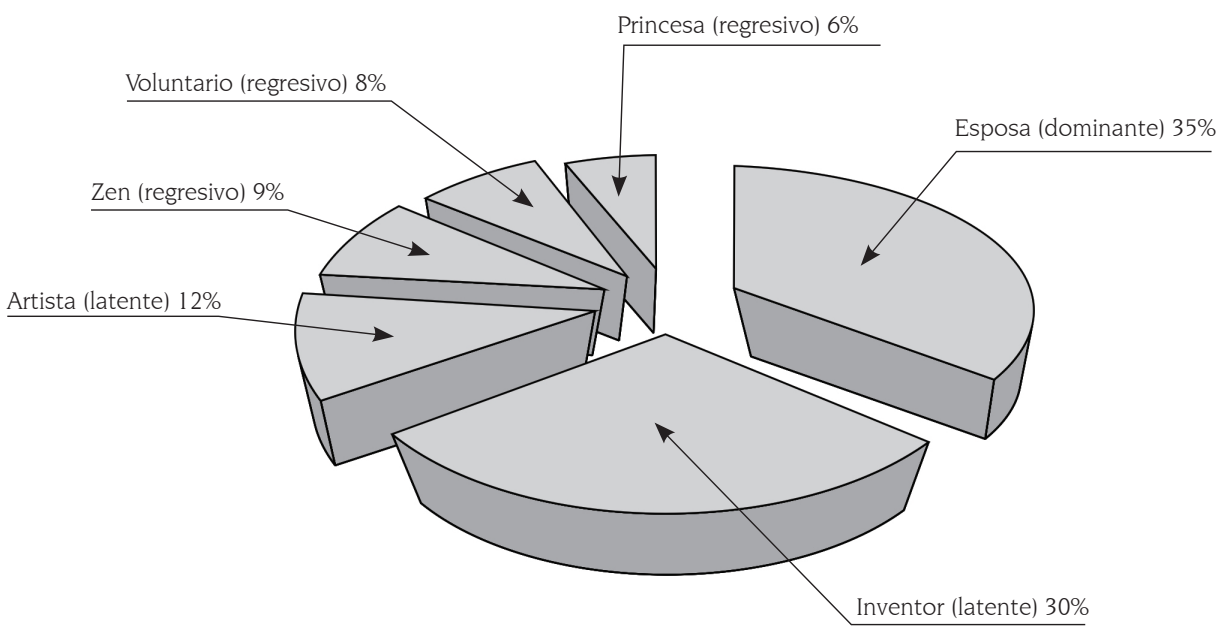

En el caso de NIKE podemos observar que los arquetipos considerados significativos son los que el $X^{2}(p)$ es menor o igual a 0,005.

Tabla 7. chi-cuadrado y t de Student de la marca NIKE. Resumen de los datos de contraste de hipótesis obtenidos a partir de la información aportada por los sujetos experimentales. El nivel de significación utilizado en todos los contrastes es para el 0,5. t de Student y 0,05 para el chi-cuadrado.

\begin{tabular}{|l|c|c|c|c|}
\hline \multicolumn{7}{|c|}{ Nike } \\
\hline \multicolumn{1}{|c|}{ Arquetipo } & \multicolumn{2}{|c|}{ Chi-cuadrado } & \multicolumn{2}{c|}{ Tde Student } \\
\hline Adolescente & $\mathrm{x}^{2}=30,857$ & $\mathrm{p}=0,000$ & $\mathrm{t}=14,207$ & $\mathrm{p}=0,000$ \\
Artista & $\mathrm{x}^{2}=3,428$ & $\mathrm{p}=0,064$ & $\mathrm{t}=11,454$ & $\mathrm{p}=0,000$ \\
Celebridad & $\mathrm{x}^{2}=7,714$ & $\mathrm{p}=0,005$ & $\mathrm{t}=11,137$ & $\mathrm{p}=0,000$ \\
Intelectual & $\mathrm{x}^{2}=0,857$ & $\mathrm{p}=0,354$ & $\mathrm{t}=13,863$ & $\mathrm{p}=0,000$ \\
Esposa & $\mathrm{x}^{2}=1,523$ & $\mathrm{p}=0,217$ & $\mathrm{t}=14,287$ & $\mathrm{p}=0,000$ \\
Explorador & $\mathrm{x}^{2}=11,523$ & $\mathrm{p}=0,000$ & $\mathrm{t}=11,096$ & $\mathrm{p}=0,000$ \\
Madre & $\mathrm{x}^{2}=7,714$ & $\mathrm{p}=0,005$ & $\mathrm{t}=17,211$ & $\mathrm{p}=0,000$ \\
Héroe & $\mathrm{x}^{2}=21,428$ & $\mathrm{p}=0,000$ & $\mathrm{t}=11,763$ & $\mathrm{p}=0,000$ \\
Inventor & $\mathrm{x}^{2}=13,714$ & $\mathrm{p}=0,000$ & $\mathrm{t}=11,146$ & $\mathrm{p}=0,000$ \\
Patriarca & $\mathrm{x}^{2}=0,380$ & $\mathrm{p}=0,537$ & $\mathrm{t}=13,477$ & $\mathrm{p}=0,000$ \\
Niño & $\mathrm{x}^{2}=13,714$ & $\mathrm{p}=0,000$ & $\mathrm{t}=11,146$ & $\mathrm{p}=0,000$ \\
Princesa & $\mathrm{x}^{2}=18,666$ & $\mathrm{p}=0,000$ & $\mathrm{t}=22,909$ & $\mathrm{p}=0,000$ \\
Príncipe & $\mathrm{x}^{2}=9,523$ & $\mathrm{p}=0,002$ & $\mathrm{t}=11,096$ & $\mathrm{p}=0,000$ \\
Voluntario & $\mathrm{x}^{2}=16,095$ & $\mathrm{p}=0,000$ & $\mathrm{t}=21,354$ & $\mathrm{p}=0,000$ \\
Zen & $\mathrm{x}^{2}=21,428$ & $\mathrm{p}=0,000$ & $\mathrm{t}=24,834$ & $\mathrm{p}=0,000$ \\
\hline
\end{tabular}

Fuente: Sabrine Dornelles, GRP 2010 
Según la tabla 7 podemos observar que el chicuadrado es menor o igual a 0,05 en los arquetipos adolescente, celebridad, explorador, madre héroe, inventor, niño, princesa, príncipe, voluntario y zen. Los demás arquetipos no parecen ser significativo, ya que posee un $\mathrm{x}^{2}$ más grande que 0,05.

La t de Student, también, da un valor significativo de 0,00 para todos los arquetipos, es decir, para todas las variables; esto quiere decir en este caso, que todos los arquetipos son significativos. Así que en este caso, utilizaremos los resultados de la $\mathrm{x}^{2}$ para considerar las variables que son significativos o no. Y la t de Student solamente utilizaremos como una referencia que reafirma los valores del chi-cuadrado.

Como hemos mostrado en la tabla 7 los arquetipos significativos en la marca Nike son los arquetipos adolescente, celebridad, explorador, madre héroe, inventor, niño, princesa, príncipe, voluntario y zen. Partiendo de estos arquetipos significativos encontrados en la marca NIKE con los valores del chi-cuadrado, determinaremos los arquetipos dominantes y regresivos utilizando la frecuencia y el porcentaje.
Repetiremos el análisis con la marca Puma; primeramente analizaremos el chi-cuadrado correspondiente a cada una de los arquetipos relacionados a la marca Puma. También analizaremos el t de Student utilizando para ello el valor $\mathrm{p} \leq 0,5$ para que sea una variable significativa y considerando que el valor de chi-cuadrado debe ser $p \leq 0,05$. Como podemos observar, los arquetipos considerados significativos en el caso de PUMA son los que el X² (p) es menor o igual a 0,005.

Según la tabla 8 podemos observar que el chi-cuadrado es menor o igual a 0,05 en los arquetipos, princesa, príncipe voluntario y zen. Los demás arquetipos no parecen ser significativos, ya que poseen un $\mathrm{x}^{2}$ más grande que 0,05.

La t de Student, también, da un valor significativo de 0,00 para todos los arquetipos, es decir, para todas las variables; esto quiere decir que todos los arquetipos son significativos. Así que en este caso, utilizaremos los resultados de la $\mathrm{x}^{2}$ para considerar las variables que son significativos o no. Y la t de Student solamente la utilizaremos como una referencia que reafirma los valores del chi-cuadrado.

Tabla 8. Arquetipos dominantes y regresivos NIKE. Resumen de los datos de "este arquetipo está presente en la marca Nike" obtenidos a partir de la información aportada por los sujetos experimentales.

\begin{tabular}{|l|c|c|c|l|}
\hline \multicolumn{5}{|c|}{ Nike } \\
\hline \multicolumn{1}{|c|}{ Arquetipos } & $x 2$ & Frecuencia (SI) & $\%$ & Clasificación \\
\hline Adolescente & $\mathrm{p}=0,000$ & 39 & 92,9 & Dominante \\
Héroe & $\mathrm{p}=0,000$ & 36 & 85,7 & Dominante \\
Inventor & $\mathrm{p}=0,000$ & 33 & 78,6 & Dominante \\
Niño & $\mathrm{p}=0,000$ & 33 & 78,6 & Dominante \\
Explorador & $\mathrm{p}=0,000$ & 32 & 76,2 & Dominante \\
Príncipe & $\mathrm{p}=0,002$ & 31 & 73,8 & Latente \\
Celebridad & $\mathrm{p}=0,005$ & 30 & 71,4 & Latente \\
Madre & $\mathrm{p}=0,005$ & 12 & 28,6 & Latente \\
Voluntario & $\mathrm{p}=0,000$ & 8 & 19 & Regresivo \\
Princesa & $\mathrm{p}=0,000$ & 7 & 16,7 & Regresivo \\
Zen & $\mathrm{p}=0,000$ & 6 & 14,3 & Regresivo \\
\hline
\end{tabular}

Fuente: Sabrine Dornelles, GRP 2010 
Gráfica 5. Arquetipos Dominantes, Latentes y Regresivos de NIKE

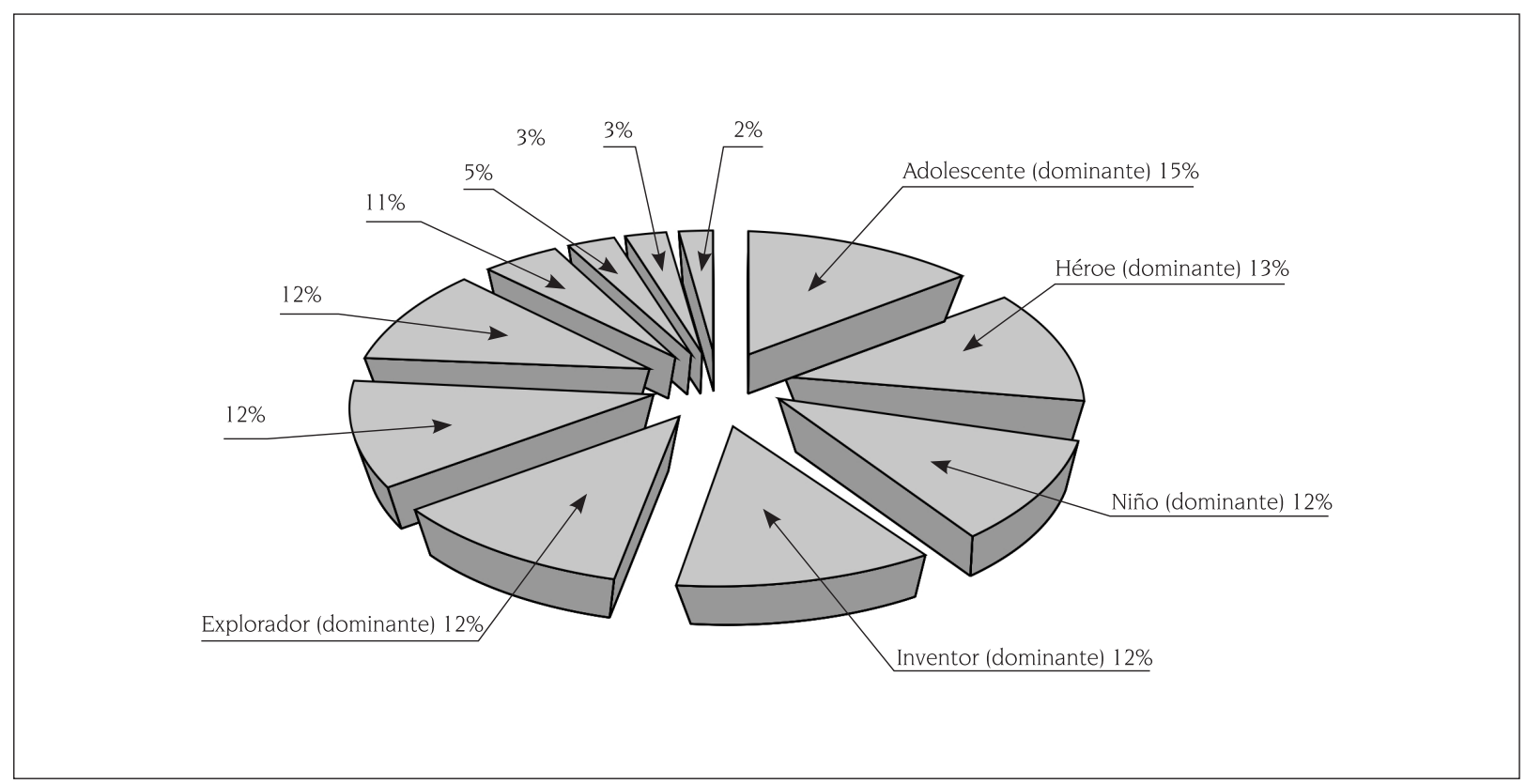

Como hemos observado en la tabla 8 los arquetipos significativos en la marca PUMA son los arquetipos adolescente, princesa, príncipe voluntario y zen. Partiendo de estos arquetipos significativos encontrados en la marca Puma con los valores del chi-cuadrado y t de Student, también determinaremos los arquetipos dominantes y regresivos utilizando la frecuencia y el porcentaje.

Tabla 9. Arquetipos Dominantes y Regresivos Puma.

Resumen de los datos de "estos arquetipo está presente en la marca Puma" obtenidos a partir de la información aportada por los sujetos experimentales.

\begin{tabular}{|l|c|c|c|l|}
\hline \multicolumn{5}{|c|}{ Puma } \\
\hline Arquetipos & X2 & $\begin{array}{c}\text { Fre- } \\
\text { cuen- } \\
\text { cia (si) }\end{array}$ & $\%$ & Clasificación \\
\hline Principe & $\mathrm{p}=0,000$ & 37 & 88,1 & Dominante \\
\hline Adolescente & $\mathrm{p}=0,005$ & 30 & 71,4 & Latente \\
\hline Zen & $\mathrm{p}=0,001$ & 10 & 23,8 & Regresivo \\
\hline Princesa & $\mathrm{p}=0,000$ & 7 & 16,7 & Regresivo \\
\hline
\end{tabular}

Fuente: Sabrine Dornelles, GRP 2010
Una vez definidos los arquetipos dominante, latente y regresivo de cada una de las marca podemos definir el Perfil arquetípico de la marca utilizando los arquetipos Dominantes y Latentes.

El perfil arquetípico de la marca significa que la marca posee las mismas características que los arquetipos dominantes y latentes que la constituyen, es decir, las características presentes en los arquetipos que constituyen el perfil arquetípico son las mismas características presentes en la propia marca, o son las características que los sujetos perciben en la marca.

Llegados a este punto, estamos en condiciones de valorar las 4 hipótesis que se dieron al iniciar este trabajo.

a) Hipótesis 1: Existe relación entre los arquetipos y las marcas

Según los resultados de la investigación la hipótesis 1 debe ser considerada válida, pues los sujetos han identificado relación entre las 
Gráfica 6. Arquetipos Dominantes, Latentes y Regresivos de Puma

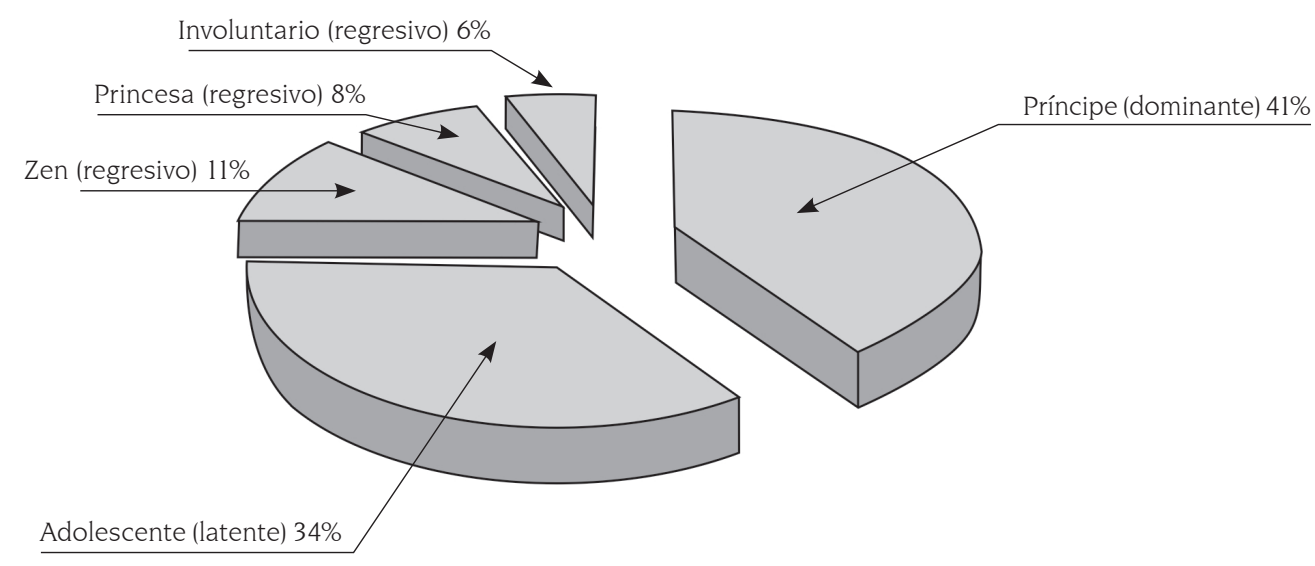

Fuente: Sabrine Dornelles, GRP 2010

marcas y los arquetipos. O sea los entrevistados han afirmado ver características de los arquetipos con las tres marcas estudiadas: ADIDAS, NIKE Y PUMA. Si analizamos las tablas anteriores, donde se indican los valores del chi-cuadrado para cada marca, podemos observar que hay significación entre las marcas y algunos arquetipos.

b) Hipótesis 2: Podemos identificar al menos un arquetipo en las marcas convencionales

Analizando todos los datos obtenidos también damos por válida la hipótesis 2. Y lo hacemos dado que sí que hemos identificado al menos un arquetipo en cada una de las marcas deportivas estudiadas. Utilizamos las frecuencias y porcentajes para definir los arquetipos dominantes significativos para contrastar esta hipótesis. Observamos que en cada una de las marcas hemos encontrado al menos un arquetipo significativo dominante.

c) Hipótesis 3: Podemos identificar más de un arquetipo en las marcas convencionales

La hipótesis 3 también es considerada válida. Ya que en las marcas estudiadas hemos encontrado como mínimo dos arquetipos (entre
Dominantes y Latentes) relacionados con cada una de las marcas.

Tabla 10. Arquetipos presentes en las marcas Adidas, Nike y Puma.

\begin{tabular}{|l|l|l|l|}
\hline & Adidas & Nike & Puma \\
\hline Arquetipos & Esposa & Adolescente & Adolescente \\
Dominantes & Inventor & Celebridad & Príncipe \\
Latentes & Artista & Explorador & \\
& & Héroe & \\
& & Inventor & \\
& Niño & \\
& & Principe & \\
& & Madre & \\
\hline
\end{tabular}

Fuente: Sabrine Dornelles, GRP 2010

d) Hipótesis 4: Podemos identificar el perfil arquetípico de las marcas utilizando los arquetipos encontrados en ella

La hipótesis 4 también es considerada válida, ya que partiendo de los datos analizados de las encuestas hemos identificado el porcentaje de frecuencia de cada arquetipo dominante y latente en la marca. Partiendo de esto hemos podido diseñar el Perfil arquetípico de cada una de las marcas. 
Gráfico 7. Perfil Arquetípico de Adidas

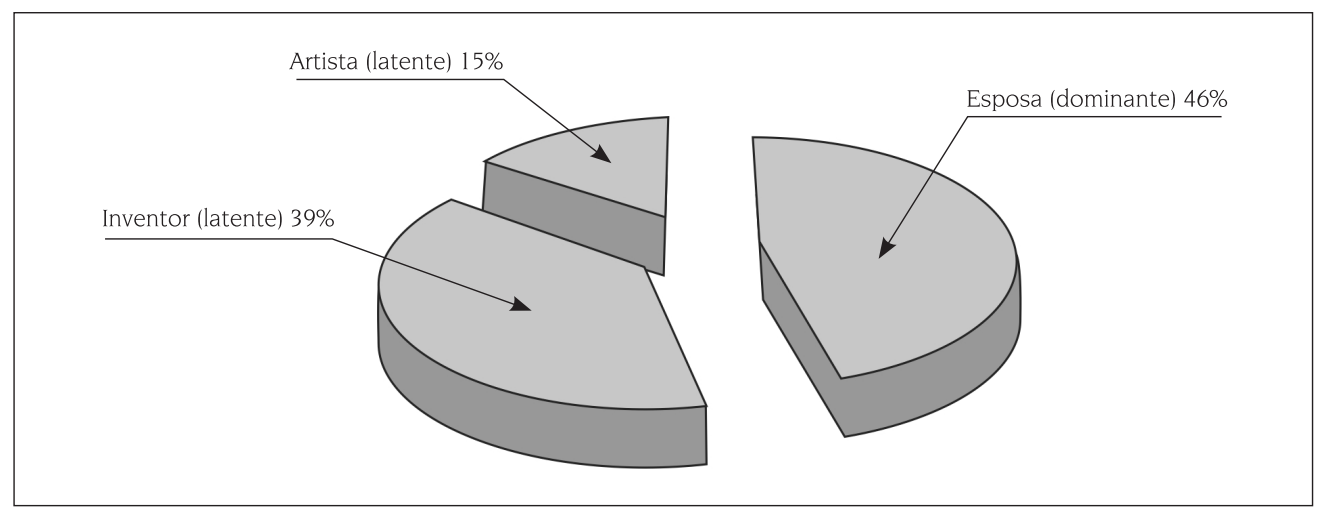

Fuente: Sabrine Dornelles, GRP 2010

Gráfica 8. Perfil Arquetípico de Nike

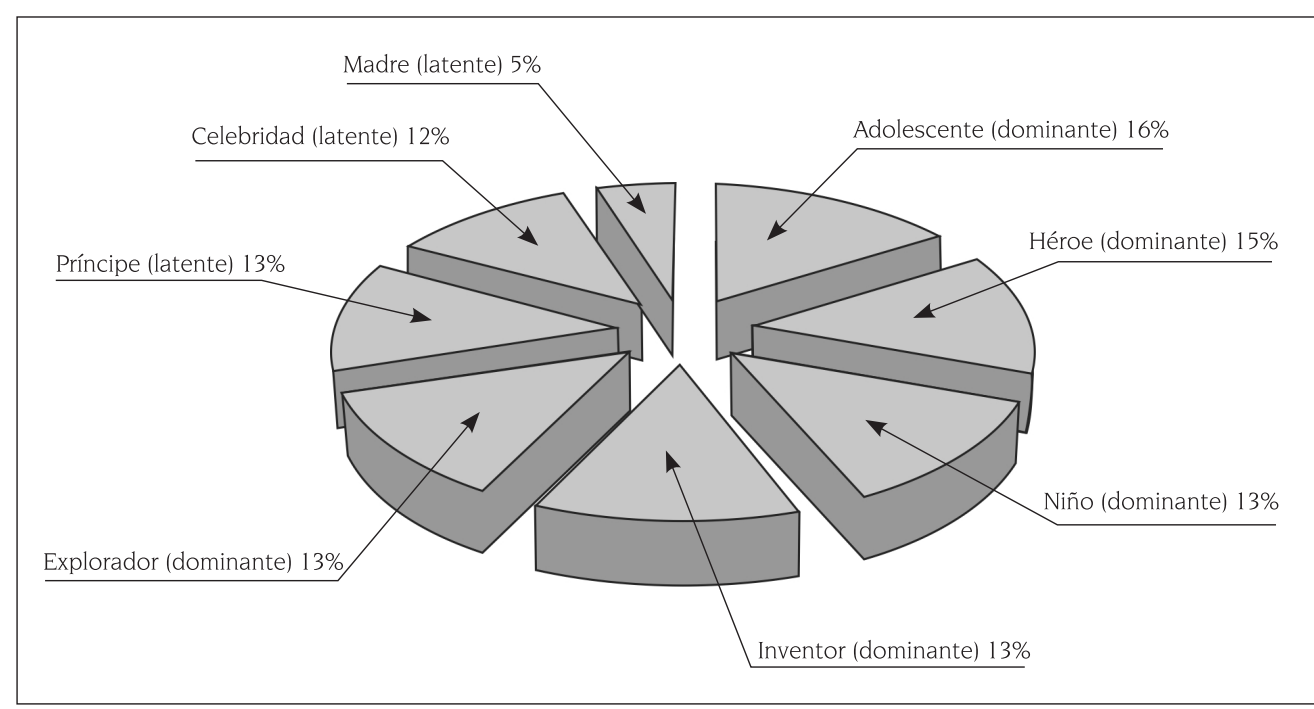

Fuente: Sabrine Dornelles, GRP 2010

Gráfica 9. Perfil Arquetípico de Puma

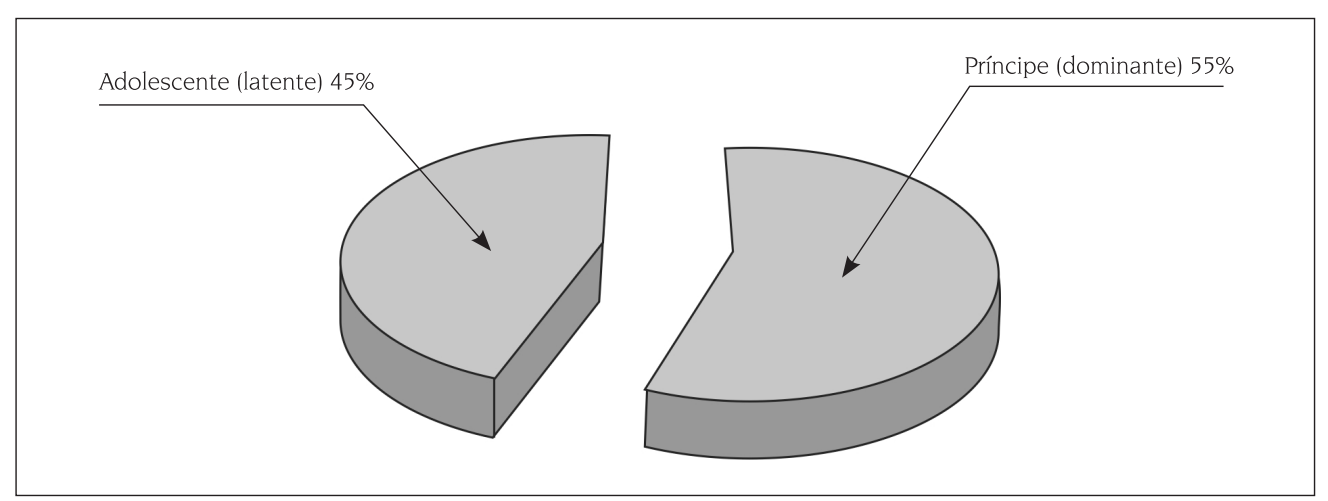

Fuente: Sabrine Dornelles, GRP 2010

Anagramas, Volumen 10, № 19, pp. 89-108 


\section{Conclusiones}

En este trabajo hemos logrado mezclar dos temas que aparentemente puede parecer que no tienen ninguna relación: las marcas y los arquetipos.

Es como afirma la autora Vázquez (2007: 98): "Aunque estamos muy condicionados por el pensamiento científico racional, podemos afirmar que la ciencia también es temporal, ya que cada vez se desarrolla con más rapidez; ésta es histórica y no puede monopolizar el conocimiento más que por un tiempo determinado. No aceptar el mítico es renunciar a profundizar en nuestra naturaleza y el hombre actual debe reconocerla para saber qué lugar ocupa en el mundo y cuál es el sentido de su existencia a partir de los fenómenos tan complejos que conforman la psique. En realidad, los mitos nos llevan por un camino cuyo fin será conocernos mejor..."

En el presente trabajo entendemos que la gestión de la marca ha pasado a convertirse en una cuestión de primer orden en la estrategia de las organizaciones. Elementos como la identidad e imagen de la marca definen la capacidad de relación de la misma con los clientes, estableciendo su potencial para crear, mantener y consolidar la relación con los consumidores. Y, pese a las dificultades y limitaciones para controlar estos elementos, se busca, en la medida de lo posible, desarrollar actuaciones encaminadas a fortalecer en la imagen de la marca aquellos aspectos de la identidad, más valorados, que entre otras, cobran especial importancia en el desarrollo de la política de imagen de marca.

Esta importancia comunicativa se basa en el establecimiento de una adecuada identidad de marca, así como la correcta conversión de la misma en una imagen de marca que permita a la organización diferenciarse del resto de los competidores y lograr cubrir un hueco en el mercado. La identidad de marca es el paso inicial que ha de seguir la empresa para llegar a construir una correcta imagen. A partir de esta imagen de marca el consumidor establece un posicionamiento del producto en relación con la competencia, y es esta la base competitiva que va permitir a la empresa, diferenciarse y sobrevivir a lo mercado tan complejo como el actual. Es necesario trabajar con una personalidad atractiva, diferente, llena de significados para los clientes potenciales y conectar en un nivel emocional con la marca, dotándola de un valor añadido diferente. En definitiva, es un proceso mucho más complejo que se llama estrategia de marca y que consiste en dar a la marca el poder de atracción y significados relevantes e incuestionables que consigan un estar en la mente de los públicos.

Así que desde este trabajo se aporta algunas reflexiones sobre lo estudiado:

- Las organizaciones construyen una marca fuerte agregando valores que son percibidos por los públicos;

- Estos valores no son solo de calidad, confiabilidad y precio, sino que se trata también, de un conjunto de atributos y significados, en gran parte social, moral y emocional.

- La marca se comunica a través de estos valores, creando una relación con los públicos;

- Estos valores deben plasmarse en la identidad de la marca y serán percibidos por los públicos a través de la imagen de la marca.

- La percepción es la imagen que los públicos tienen de la marca. Es la personalidad de la marca que está en la mente del consumidor.

- Así como las personas tienen personalidad, las marcas también posee una personalidad, que está constituida por diferentes arquetipos que están en el inconsciente colectivo de todas las personas. 
Para entender mejor esta conexión entre marca y consumidor a través de los arquetipos, ha sido necesaria una reflexión profunda sobre los arquetipos. Hemos definido 15 arquetipos para relacionarlos con las marcas. Hemos partido de los arquetipos de los dioses griegos estudiados y definidos por la psicología como formas del comportamiento humano.

Ha sido así como hemos concluido que:

- los arquetipos son elementos simbólicos.

- los arquetipos están presentes en el inconsciente colectivo de las personas.

- el arquetipo puede ser estudiado a través de los mitos, sueños y epopeyas.

- utilizando la mitología griega se pueden encontrar quince arquetipos muy bien definidos.

Los atributos de los arquetipos de los quince dioses griegos estudiados han sido identificados y utilizados en relación con su presencia en nuestro inconsciente colectivo:

Diosa Afrodita - Celebridad - Es el arquetipo de la sensualidad, del glamor y de la seducción.

Dios Apolo - Príncipe - Es el arquetipo de la belleza estética, de la forma, del equilibrio.

Dios Ares - Héroe - Es el arquetipo de la fuerza, de la superación, de la competición con el único objetivo la victoria.

Diosa Artemisa - Explorador - Es el arquetipo de la expansión, del movimiento, la independencia y la libertad.

Diosa Atenas - Intelectual - Es el arquetipo del estratega, de la objetividad y lo racional

Diosa Deméter - Madre - Es el arquetipo de la madre, de la generosidad, de la progenitora que ama y protege y acoge.
Dios Dionisio - Adolescente - Es el arquetipo del joven de la rebeldía, del adolecente que busca romper con los patrones de la sociedad y de sus padres, que busca nos amigos su mundo y su tribu.

Dios Hades- Zen - Es el arquetipo del contacto interior, con la naturaleza y con la vida serena, pura, sencilla, comer sano y encontrar su interior en la soledad.

Dios Hefesto - Inventor - Es el arquetipo del que crea materiales funcionales, innovadores y creativos.

Diosa Hera - Esposa - Es el arquetipo de la tradición, del compromiso en la familia, del conservadurismo.

Dios Hermes - Niño - Es el arquetipo de la travesura, del lúdico, mágico, niñez.

Diosa Hestia - Voluntario - Es el arquetipo de la ayuda al próximo, de la unidad del mundo, todos somos hermanos y tenemos que ayudarnos.

Diosa Perséfone - Princesa - Es el arquetipo de la inocencia, de los cuentos de hadas, del romántico.

Dios Poseidón - Artista - Es el arquetipo de las emociones expresadas con el arte, de la artesanía, una explosión de sensibilidad y pasión.

Zeus - Patriarca - Es el arquetipo del Poder, del padre, de la autoridad, del rey.

Con los datos obtenidos en esta investigación podemos comprobar que no solo existe una relación profunda entre las Marcas y los Arquetipos sino que también se puede comprender el fenómeno de las marcas utilizando el perfil arquetípico de las marcas.

A partir de la imagen de marca, el consumidor establece un posicionamiento y la relación 
con los arquetipos presentes en las marcas son la conexión que une al consumidor y las marcas. Batey (2008:37) afirma que las personas son multifacéticas, es decir, poseen todos o gran parte de los arquetipos dentro de su inconsciente; los arquetipos se manifiestan según la necesidad, dependiendo de lo que uno necesita en cada momento. La naturaleza de la relación arquetípica entre la marca y los consumidores es que la marca personifica el significado del arquetipo para los públicos de la marca.

Al ser capaces de reconocer esos arquetipos y asociarlos a determinados valores universales, los hace tremendamente interesantes para la construcción de la personalidad de la marca. Lo que afirmamos en este trabajo es que es posible identificar los arquetipos en las marcas lo que nos permite construir su perfil arquetípico.

Hemos podido validar las hipótesis planteadas al principio de la investigación y aunque solamente las marcas ADIDAS, NIKE y PUMA han sido analizadas en este trabajo, se intuye su aplicabilidad a las demás marcas convencionales. Y por tanto podemos pensar en la descripción de los perfiles arquetípicos de las Marcas para cualquier marca. Así los consultores, brand managers, publicistas y el resto de profesionales de marketing, publicidad y relaciones públicas podrán tener más precisión, claridad y coherencia en los discursos generados por la marca.

\section{Bibliografía}

ATKIN, Douglas. El culto a las marcas.2.ed. Barcelona: Robinbook, 2005.

BATEY, Mark. Brand Meaning. New York; Routledge, 2008.

BOLEN, Jean Shinoda. Las Diosas de cada Mujer. Una nueva psicología femenina. 13 ed. Barcelona: kairós, 2006.

BOLEN, Jean Shinoda. Los Dioses de cada Hombre. Una nueva psicología masculina. Barcelona: Kairós, 1999.

Bonilla, V. E. Confiabilidad, en el boletín informativo INEVA en acción, Vol. 2, No. 3, pp.1-3.

BLACKETT, Tom; BOAD, Bob. Co-branding: la ciencia de la alianza. Buenos Aires: Pearson Educación, 2001.

CLIFTON, Rita: SIMMONS, John. Brands and Branding. London: The Economist, 2003.

CLIFTON, Rita. La Imagen de Marca.1 ed. Barcelona: Paidós Iberica, 2004.

CLIFTON, Rita y Esther Maughan, Twenty-Five Visions: The Future of Brands. New York: University Press Nueva York, 2000.

DORNELLES, Sabrine, El perfil arquetípico de las marcas. Tesis Doctoral. Universidad Autónoma de Barcelona, 2010.

GEORGE, D. \& MALLERY, P. SPSS/PC+ step by step: A simple guide and reference. Wadsworth Publishing Company. Belmont, CA: Estados Unidos, 1995.

HERNÁNDEZ, Roberto Sampieri; FERNÁNDEZ, Carlos Collado; BAPTISTA, Pilar Lucio. Metodología de la Investigación. México: Mc Graw Hill, 2003. I

IGARTUA, Juan José; HUMANES, Maria Luisa: Teoría e investigación en comunicación social. Madrid: Síntesis, D.L., 2004.

IGARTUA, Juan José Perosanz. Métodos Cuantitativos 
de Investigación en comunicación. Barcelona: Bosch, 2006.

JUNG, Carl Gustav. Arquetipos e inconsciente colectivo. 4. ed. Barcelona: Paidós, 1991.

JUNG, Carl Gustav. El hombre y sus símbolos. $6^{\circ}$ ed. Barcelona: Caralt, 1997.

KOTLER, Philip. Fundamentos de Marketing. $6^{\mathrm{a}}$ ed. México: Pearson Educación, 2003.

KUDER, G. F. \& Richardson, M. W. The theory of the estimation of test reliability. Psychometrika: Springer New York, Volume 2, Number 3 / septiembre de 1937.

MARTINS, José. A Natureza Emocional da Marca. 2. ed. São Paulo: Negócio Editora 1999.
MARTINS, José Roberto. Branding. 1 ed. São Paulo:Negócio Editora, 2000.

MARTIN, René. MITOLOGÍA Griega y Romana. 10ª ed. Madrid: Espasa, 2006.

RAPAILLE, Clotaire. The Culture Code. Na ingenious way to understand why people aroud the world live and buy they do. New York: Broadway Books, 2006.

TENA, Daniel Parera. Diseño Grafico y Comunicación. led. Madrid: Pearson Prentice-Hall, 2005.

Vázquez, B. (2007). Publicidad Emocional. Estrategias creativas. Madrid: ESIC Editorial. 
